\title{
Wege zu verantwortungsvoller Forschung und Entwicklung im Bereich der Pflegerobotik: Die ambivalente Rolle der Ethik
}

\author{
Christoph Kehl
}

\begin{abstract}
Zusammenfassung
Obwohl die Entwicklungsarbeiten an Pflegerobotern bis in die 1980er Jahre zurückreichen, waren die bisherigen Bemühungen nicht von größeren Erfolgen gekrönt. Zwar wurde eine Vielzahl an Produktvisionen und Prototypen hervorgebracht, diese haben jedoch in aller Regel noch nicht den Weg in den Pflegemarkt gefunden. Dies hängt nicht nur mit technischen Schwierigkeiten zusammen, sondern lässt sich auch auf die bislang stark technikfokussierte Ausrichtung der Forschungs- und Entwicklungspraxis zurückführen: Offenbar ist es noch nicht gelungen, Angebote zu entwickeln, die von Kostenträgern und Endkunden ausreichend akzeptiert werden. Vor diesem Hintergrund geht der Beitrag der Frage nach, wie sich eine verantwortungsvolle Forschungs- und Entwicklungspraxis gestalten lässt, die den hohen Anforderungen an Serviceroboter-Anwendungen im Pflegebereich gerecht wird. Mit Blick auf neuere politische Konzepte zur Technology Governance wird dargelegt, dass neben einer bedarfsorientierten Vorgehensweise vor allem der angemessene Umgang mit normativen Fragen und Unsicherheiten heraussticht, was auf die zentrale Bedeutung ethischer Reflexion verweist. Die Schwierigkeiten, die sich bei der ethischen Bewertung der robotischen Pflegeanwendungen ergeben, werden beleuchtet, und es wird schließlich die ambivalente Rolle der Ethik bei der Governance der Pflegerobotik herausgearbeitet.
\end{abstract}

Erste Entwicklungsarbeiten an robotischen Helfern für den Gesundheits- und Pflegebereich lassen sich bis in die 1980er Jahre zurückverfolgen. HelpMate, ein autonomer

C. Kehl $(\square)$

Büro für Technikfolgen-Abschätzung beim Deutschen Bundestag (TAB),

Berlin, Deutschland

E-Mail:kehl@tab-beim-bundestag.de

(C) Der/die Autor(en) 2018

O. Bendel (Hrsg.), Pflegeroboter, https://doi.org/10.1007/978-3-658-22698-5_8 
Transportroboter für Krankenhäuser, wurde Anfang der 1990er Jahre vorgestellt (Evans 1994); etwa zur selben Zeit entstand Handy 1, eine Esshilfe für zerebral Gelähmte (Topping 2000). Mehr als 25 Jahre danach ist zu konstatieren, dass zwar die Entwicklungsbemühungen deutlich zugenommen haben - was sich in einer breiten Palette an unterschiedlichsten Prototypen widerspiegelt -, größere Markterfolge aber immer noch auf sich warten lassen. Die wenigen Systeme, die den Weg in die Pflegepraxis gefunden haben, werden meist nur in geringer Stückzahl vertrieben. Zu den bekanntesten gehört sicherlich die Roboterrobbe Paro, die in Europa seit 2009 zugelassen ist und in Deutschland in rund 50 Pflegeheimen eingesetzt wird. Bemerkenswert ist, dass Paro ursprünglich als Spielzeug entwickelt wurde und in Japan bei Privatpersonen offenbar auf fast größeres Interesse zu stoßen scheint als bei Pflegeeinrichtungen (Shibata 2012). Ähnliches gilt für den Assistenzroboter Care-O-bot, der seit den 1990er Jahren vom Fraunhofer-Institut für Produktionstechnik und Automatisierung (IPA) entwickelt wird. Das System wurde vor allem mit Blick auf die Unterstützung häuslicher Pflegetätigkeiten entwickelt und diesbezüglich in verschiedenen Anwendungsszenarien getestet (z. B. als Butler für Hol- und Bringdienste, als Kommunikationsplattform oder zur Sturzerkennung). Die vierte Generation des Haushaltsassistenten, die 2015 vorgestellt wurde, soll nun endlich „die Basis für kommerzielle Serviceroboter-Lösungen“ bieten (Fraunhofer IPA 2015). Dabei steht jedoch weniger die Pflege im Vordergrund, sondern der Einzelhandels- und Entertainmentbereich. So wird der Roboter unter dem Namen Paul ohne Arme als ,mobiler Informationskiosk“ etwa bereits erfolgreich zur Kundenführung in Elektronikmärkten eingesetzt. Ob und wann Care-O-Bot in der Pflege routinemäßig zum Einsatz kommen wird, ist derzeit völlig unklar.

Wie lässt sich diese auffällige Diskrepanz zwischen Aufwand und Ertrag erklären? Dass autonome Pflegeroboter in der Regel von einer hohen technischen Komplexität sind und die Entwicklungsprozesse entsprechend aufwendig, ist sicherlich richtig, taugt jedoch als Antwort nur bedingt. Schließlich zeichnen sich gerade die am Markt verfügbaren Pflegeroboter wie die Robbe Paro durch ein technisches Niveau aus, das in etwa mit dem von Staubsaugerrobotern vergleichbar ist. Dass deren Verkaufszahlen in den Millionen liegen, die von Paro jedoch bei weitem nicht, dürfte mithin kaum rein technisch erklärbar sein, sondern vielmehr damit zu tun haben, dass mit Staubsaugerrobotern ein Nutzen geschaffen wird, den Pflegeroboter bislang verfehlt haben. Der 7. Altenbericht der Bundesregierung hält mit Blick auf technische Assistenzsysteme entsprechend fest, dass es noch nicht gelungen sei, Angebote für die Pflege zu entwickeln, die von Kostenträgern und Endkunden ausreichend akzeptiert werden (Bundesregierung 2016, S. 253). Auch TA-Experten haben immer wieder auf diesen Umstand hingewiesen und ihn u. a. dadurch erklärt, dass die bisherigen Innovationspfade allzu stark einem technischen Imperativ verhaftet waren (Krings et al. 2012). Demzufolge waren es also primär technologische Machbarkeitsvisionen, welche Forschung und Entwicklung vorangetrieben haben, weniger jedoch die tatsächlichen Problem- und Bedürfnislagen der relevanten Nutzergruppen. Das Resultat seien Artefakte, welche zwar technisch durchaus gelungen sein mögen, jedoch die komplexen Anforderungen an Pflege nicht angemessen erfüllen - angesichts der Herausforderungen, die der demografische Wandel für die Pflege bereithält, eine durchaus ernüchternde Diagnose. 
Vor diesem Hintergrund wird in diesem Beitrag der Frage nachgegangen, wie sich eine Forschungs- und Entwicklungspraxis gestalten lässt, die den hohen Anforderungen an Serviceroboter-Anwendungen im Pflegebereich gerecht wird. Mit Blick auf neuere politische Konzepte zur verantwortungsvollen Technikgestaltung wird dargelegt, dass neben einer bedarfsorientierten Vorgehensweise dabei vor allem der angemessene Umgang mit normativen Fragen und Unsicherheiten heraussticht, die auf die zentrale, jedoch durchaus zwiespältige Rolle ethischer Reflexion verweisen.

\subsection{Bedarfe und Bedürfnisse im Fokus}

Bei der Pflege handelt es sich um einen stark ausdifferenzierten Dienstleistungsbereich. Grundsätzlich zu unterscheiden sind die ambulante Pflege zu Hause sowie die stationäre Pflege im Heim, die wiederum sehr verschiedene Tätigkeiten umfassen (z. B. Waschen, Ernährung, Mobilisation, emotionale Zuwendung). Hinzu kommt, dass man es mit sehr unterschiedlichen Zielgruppen zu tun hat, was zu einer Separierung unterschiedlicher Pflegeberufe geführt hat (Kinderpflege, Altenpflege, Krankenpflege etc.). Angesichts des demografischen Wandels werden in der Altenpflege die größten Potenziale für robotische Lösungen gesehen - gerade in dem Bereich also, in dem die Spannbreite an Beeinträchtigung resp. Pflegebedürftigkeit besonders ausgeprägt ist. Sie reicht von älteren Menschen, die noch zu Hause wohnen, dabei aber Unterstützung benötigen, über Heimbewohner, die unterschiedliche Grade und Formen der Gebrechlichkeit aufweisen, bis hin zu Demenzpatienten im fortgeschrittenen Stadium, die rund um die Uhr der Betreuung bedürfen. Als personenbezogene Dienstleistung ist Pflege gefordert, dieser Vielfältigkeit an Ansprüchen und Lebensumständen gerecht zu werden, wobei die zwischenmenschliche Begegnung sowie die fürsorgliche Zuwendung - also der ,Zugang zum Anderen“ (Hülsken-Giesler 2008) - als Dreh- und Angelpunkt pflegerischen Handelns gelten (vgl. Görres und Friesacher 2005). Da immer wieder situativ auf individuelle und sich wandelnde Bedürfnisse reagiert werden muss, ist Pflegearbeit folglich nur begrenzt standardisierbar. Dasselbe trifft dann notgedrungen auch auf die Anforderungen an technische Unterstützungslösungen zu, die sich letztlich nur dann sinnvoll in die Pflegearbeit integrieren lassen, wenn sie spezifisch auf die unterschiedlichen Pflegesituationen zugeschnitten sind. Dies ist umso mehr der Fall, als von Pflegebedürftigen aufgrund deren körperlichen und psychischen Einschränkungen nicht erwartet werden kann, dass sie zu größeren Anpassungsleistungen an neue technische Hilfsangebote in der Lage sind. Folglich wird im Pflegebereich die Nachfrage nach technischen Innovationen ,nicht primär aus dem Angebot generiert“ - wie es etwa bei Staubsaugerrobotern eindeutig der Fall ist -, sondern ergibt sich vielmehr aus ,handfesten Bedarfen, die nicht aus freien Nutzungsentscheidungen erwachsen“ (Elsbernd et al. 2015, S. 71).

Wie der Überblicksartikel von Östlund und Frennert (2014) am Beispiel der sozialen Robotik aufzeigt, ist die Perspektive älterer Menschen in Forschung und Entwicklung bisher kaum präsent gewesen, stattdessen herrschen stereotypische Sichtweisen der 
Bedürfnisse dieser Personengruppe vor. Für andere Robotertypen dürfte Ähnliches gelten. Vor diesem Hintergrund ist der Ruf nach einer Technikentwicklung, die sich primär an „handfesten Bedarfen“ orientiert (und weniger am technisch Umsetzbaren oder ökonomisch Vorteilhaften), in den letzten Jahren immer lauter geworden. Angemahnt wird nichts weniger als ein „Paradigmenwechsel“ hin zu einer Forschungs- und Entwicklungsperspektive, welche ,die Bedürfnis- und Bedarfslagen von pflegebedürftigen Menschen und deren Bezugspersonen“ in den Mittelpunkt der technischen Entwicklung stellt (Elsbernd et al. 2014, S. 12). Für die Pflegerobotik erscheint dies besonders drängend, da man es hierbei mit einer Technologie zu tun hat, die aufgrund ihrer Interaktivität und zunehmenden Autonomiefähigkeit ganz neue technische Möglichkeiten bietet, die aber gerade nicht Selbstzweck sein dürfen, sondern die es sinnvoll - und zwar im Sinne guter Pflege - nutzbar zu machen gilt. Wie dieser Anspruch methodisch einzulösen ist, ist noch in vielerlei Hinsicht offen. Zwar ist es in etlichen Technikbereichen längst etabliert, Aspekte der Usability frühzeitig aufzugreifen, im Feld der Pflegerobotik betritt man diesbezüglich jedoch weitgehend Neuland. Die Anforderungen sind hier auch besonders hoch: Nicht nur die potenziell große Eingriffstiefe der Servicerobotik, auch die Komplexität der Pflegearrangements mit ihren vielen involvierten Akteuren (Pflegebedürftige, Angehörige, professionell Pflegende, Dienstleister etc.) stellen hohe Ansprüche an eine bedarfsorientierte Vorgehensweise. Eines der ersten Projekte, das sich mit Umsetzungsmöglichkeiten befasste, ist WiMi-Care („Förderung des Wissenstransfers für eine aktive Mitgestaltung des Pflegesektors durch Mikrosystemtechnik“), das von 2008 bis 2011 durchgeführt wurde. ${ }^{1}$ Die dort entwickelten Verfahren sind inzwischen in diversen anderen Entwicklungsprojekten erprobt und weiterentwickelt worden, beruhend auf folgenden Kernelementen (vgl. Derpmann und Compagna 2009; Kehl 2018, S. 161 ff.):

1. Interdisziplinäre Bedarfserhebung: Eine bedarfsorientierte Technikentwicklung setzt idealerweise direkt bei der Erhebung der Bedarfe an, ohne dass vorab irgendwelche technischen Festlegungen (etwa hinsichtlich Art oder Design der zu entwickelnden Geräte) getroffen werden. Zuallererst geht es also darum, ein genaues Verständnis für die tatsächlichen (und nicht nur unterstellten) Lebens- und Problemlagen der potenziellen Nutzer zu entwickeln. In der Regel greift man dabei auf Methoden der qualitativen Sozialforschung zurück (teilnehmende Beobachtung, Einzel- sowie Gruppeninterviews). Auf Basis einer gründlichen Beschreibung der bestehenden

\footnotetext{
${ }^{1}$ Ziel dieses vom Bundesministerium für Bildung und Forschung geförderten Verbundvorhabens war die Erprobung sinnvoller, d. h. bedarfsgerechter Unterstützungsmöglichkeiten durch die Servicerobotik im stationären Pflegekontext (https://www.uni-due.de/wimi-care/). Dazu wurde u. a. Care-O-bot 3 in zwei Einsatzszenarien getestet: als mobiler Butler sowie als Unterhaltungsplattform. Im Vordergrund von Wimi-Care stand die Entwicklung einer „Wissenstransferschleife“ für die interdisziplinäre Interaktion und Kommunikation, die von den Entwicklern über die Hersteller hin zu den Anwendern reicht.
} 
Pflegesituation - und zwar aus einem ganzheitlichen Blickwinkel, schließlich müssen sich neue Hilfsmittel im Gesamtkontext der Pflege bewähren (vgl. Elsbernd et al. 2015, S. 70) - können anschließend technische Unterstützungsbedarfe abgeleitet werden. Es liegt auf der Hand, dass dieses Unterfangen den technischen Blickwinkel bei Weitem sprengt, vielmehr von fundamental interdisziplinärer Art ist: Von Bedeutung ist insbesondere die sozialwissenschaftliche, arbeitswissenschaftliche und pflegewissenschaftliche Expertise.

2. Partizipative Technikgestaltung: Die Nutzerperspektive gilt es nicht nur bei der Bedarfserhebung (wie oben skizziert) zu berücksichtigen, sondern sie ist auch bei der konkreten Technikgestaltung einzubeziehen - schließlich sollen ja Lösungen resultieren, die die Bedürfnisse und Wünsche der Nutzer optimal abbilden und auf entsprechende Akzeptanz stoßen. Methodisch kann dies beispielsweise mit dem Ansatz des szenariobasierten Designs erreicht werden: Technische Einsatzszenarien, die auf Grundlage der Bedarfserhebung entwickelt wurden, werden dabei anschaulich dargestellt und mit den Nutzern abgestimmt. Die partizipative Ausrichtung der Bedarfsorientierung lässt sich konzeptionell im constructive technology assessment verorten, ein Ansatz, der von der unauflösbaren Verwobenheit technischer sowie gesellschaftlicher Entwicklungen ausgeht (Weinberger und Decker 2015, S. 37 f.).

3. Iterativer Wissenstransfer: Die bedarfsorientierte Technikentwicklung beginnt mit der Bedarfserhebung und endet bestenfalls mit einem innovativen Produkt, das zur Verbesserung der Pflege beitragen kann. Der Weg, der dahin führt, ist allerdings kein geradliniger: Neben den ermittelten Bedarfen gilt es notgedrungen auch Aspekte der technischen sowie später auch der betriebswirtschaftlichen Machbarkeit zu berücksichtigen, sodass die realisierten Lösungen über die verschiedenen Entwicklungsstufen hinweg (Produktvision, Umsetzungskonzept, Prototyp, Pilotanwendung) immer wieder neu auf ihre Bedarfsadäquanz hin zu befragen (und ggf. entsprechend anzupassen) sind. Das Resultat ist, im Idealfall zumindest, eine „Wissenstransferschleife“, in der die Perspektiven der relevanten Akteure - Nutzer, Techniker, Produktdesigner, Hersteller - iterativ miteinander verwoben sind (Derpmann und Compagna 2009, S. 20).

Die hohen methodischen Anforderungen an bedarfsorientierte Innovationsprozesse sollten deutlich geworden sein. Der Bedarfsorientierung inhärent ist zudem ihre Ergebnisoffenheit: Welche der ermittelten Bedarfe sich letztendlich technisch erfüllen lassen, ist aufgrund des iterativen Vorgehens vorab nicht planbar. Dies weist der öffentlichen Forschungsförderung insbesondere in den frühen, produktfernen Stadien eine entscheidende Rolle zu, da privatwirtschaftliche Akteure zu diesem Zeitpunkt aufgrund der verbundenen Investitionsrisiken in der Regel kaum zu größerem finanziellem Engagement bereit sind.

Insgesamt ist zu beobachten, dass die Forschungspolitik in den letzten Jahren tatsächlich partizipativen und bedarfsorientierten Ansätzen zunehmend größere Aufmerksamkeit geschenkt hat, ja mehr noch, diese sogar verstärkt in ihre Forschungsprogramme integriert. So wurde in „Horizont 2020“, dem 7. Forschungsrahmenprogramm der EU, 
das Konzept der verantwortungsvollen Forschung und Innovation (Responsible Research and Innovation, RRI) als Steuerungsinstrument zentral verankert (Schomberg 2013). Das Ziel ist, Forschung, Entwicklung und Innovation stärker auf die Lösung der großen gesellschaftlichen Herausforderungen unserer Zeit (,grand challenges“) auszurichten, zu denen auch die alternde Gesellschaft gehört, was u. a. durch eine breite und frühzeitige Stakeholder-Beteiligung erreicht werden soll. In Deutschland verfolgt das Bundesministerium für Bildung und Forschung (BMBF) mit dem Konzept der integrierten Forschung, das dem Forschungsprogramm zur Mensch-Technik-Interaktion und damit auch allen pflegerelevanten Förderaktivitäten zugrunde liegt, ähnliche Absichten: nämlich ,,[Pflege-]Technik für den Menschen zu gestalten“ sowie „die Nutzerinnen und Nutzer in die Konzeption und Entwicklung der technischen Lösungen zu integrieren“ (BMBF 2015). Was die konkrete Umsetzung angeht, sind beide Ansätze noch ziemlich unscharf und bedürfen der weiteren Konkretisierung. Gemeinsam ist ihnen die Leitidee, dass gesellschaftliche Bedarfe sowie die Bedürfnisse der Betroffenen frühzeitig im Innovationsprozess zu reflektieren sind - und zwar als zentrales regulatives Element der Technik- und Produktentwicklung -, um letztlich im Vergleich zur primär technologiegetriebenen Herangehensweise nicht nur sozial akzeptablere, sondern auch wirtschaftlich erfolgreichere Produkte zu generieren.

\subsection{Von der Notwendigkeit ethischer Reflexion - und ihren Schwierigkeiten und Grenzen}

Mit den soeben beschriebenen Governanceansätzen wird angestrebt, die Möglichkeiten der Technik zu nutzen, ,ohne die damit einhergehenden Herausforderungen zu übersehen oder unbeantwortet zu lassen“ (BMBF 2015, S. 4). Ziel ist, dafür zu sorgen, dass Forschung und Innovation sich möglichst am „gemeinsamen Guten“ (,public good“, vgl. Sutcliffe 2013, S.3) ausrichten, also in gesellschaftlich wünschenswerte Bahnen gelenkt werden. Die bloße Fokussierung auf übergreifende Bedarfe ist hierfür allerdings nicht ausreichend - nicht nur, weil diese aufgrund ihrer Abstraktheit offensichtlich nicht als sinnvoller Ausgangspunkt für konkrete Innovationsprozesse taugen (Decker und Weinberger 2015), sondern vor allem, weil es sich bei gesellschaftlichen Bedarfen (wie auch korrespondierenden individuellen Bedürfnissen) um empirische Phänomene handelt, aus denen sich keine normativen Zielvorstellungen ableiten lassen. So gibt es eine lange ökonomische Tradition, die heute noch in technisch-wirtschaftlichen Kontexten stark verbreitet ist (Elsbernd et al. 2015, S. 69), Bedarfe als Ergebnis objektivierbarer, messbarer Präferenzen zu definieren, die sich in der kaufkräftigen Nachfrage nach bestimmten Wirtschaftsgütern manifestieren (Piekenbrock 2018) - Bedarfsorientierung lässt sich demzufolge auch als Marktorientierung verstehen. Die Innovationsdynamik ökonomischen Triebkräften zu überlassen, ist jedoch gerade nicht, was mit verantwortungsvoller Forschung und Entwicklung (im Sinne oben genannter Ansätze) angestrebt wird. Vielmehr geht es darum, Innovation vorsorglich so zu steuern, dass die angestrebten Ergebnisse 
nicht nur im Einklang mit kollektiven Bedarfen, sondern auch gesellschaftlichen Werten sind. Konsequenterweise weisen deshalb sowohl die EU-Kommission als auch das BMBF in ihren jeweiligen Steuerungsansätzen der ethischen Bewertung eine zentrale Rolle im Rahmen der vorausschauenden Gestaltung soziotechnischer Innovationsprozesse zu.

\subsubsection{Ethische Bewertung von Pflegerobotern: Probleme und Herausforderungen}

„Technik und Innovation in den Rahmen eines expliziten Wertediskurses“ zu stellen (Bogner et al. 2015, S. 12), ist für den Bereich der Pflegerobotik zweifelsohne von besonderer Bedeutung. Wie bei kaum einem anderen Bereich der Servicerobotik treten hier ethische Dilemmata und Spannungen zutage (für einen Überblick vgl. Sharkey und Sharkey 2012a): Auf der einen Seite steht die Pflege aufgrund des demografischen Wandels bekanntlich vor gewaltigen Zukunftsherausforderungen. Durch die fortschreitende Überalterung der Bevölkerung wird mit einer steigenden Zahl von Pflegebedürftigen gerechnet, zugleich nimmt die Zahl der Erwerbstätigen ab, aus denen sich die Pflegekräfte rekrutieren. Der sich bereits heute manifestierende Fachkräftemangel im Bereich der Pflege droht sich laut Prognosen bis 2030 auf Hunderttausende unbesetzter Stellen auszuwachsen (Neuber-Pohl 2017), was auch den grundsätzlichen Bedarf an technischer Unterstützung drastisch ansteigen lässt. Der Servicerobotik wird diesbezüglich großes Potenzial zugeschrieben, da sie prinzipiell auch körperliche Tätigkeiten physisch assistieren kann und damit für ein weites Aufgabenspektrum zur Verfügung steht. Ob und inwiefern jedoch robotische Systeme zur Sicherstellung menschenwürdiger Pflege taugen, ist auf der anderen Seite hochumstritten. Denn Fürsorge, Empathie und Zuwendung gelten als essenzielle Bestandteile der Pflegearbeit, die als personenbezogene Dienstleistung technisch nur bedingt substituierbar ist. Befürchtet wird, dass durch die zunehmende Automatisierung von Pflegeprozessen, nicht zuletzt angetrieben durch ökonomische Zwänge, der zwischenmenschliche Kontakt als Kernelement guter Pflege zunehmend erodieren könnte (vgl. Dibelius et al. 2006). Insofern stellt sich die Frage, inwieweit der Kerngedanke der Robotik, nämlich die technische „Ersetzbarkeit menschlicher Handlungen“ (Decker 2013, S. 354), im Bereich der Pflege mit unseren moralischen Maßstäben überhaupt vereinbar ist.

Die Aussicht auf einen verbreiteten Robotereinsatz in der Pflege sorgt entsprechend, und das auch zu Recht, für große moralische Verunsicherung. „Informierung, Orientierung und Aufklärung der entsprechenden Debatten und Entscheidungsprozesse in normativer Hinsicht" (Grunwald 2013b, S. 239) erscheinen vor diesem Hintergrund als wichtige Voraussetzung dafür, um auf gesellschaftlicher Ebene ethisch verantwortbare Entscheidungen treffen zu können (vgl. Grunwald 2013a, S. 3). Insofern fällt der Technikethik (in diesem Fall speziell dem Teilbereich der Roboterethik, vgl. Loh 2017) eine wichtige gesellschaftliche Orientierungsaufgabe zu. Allerdings hat die Ethik aus 
verschiedenen Gründen Mühe, in Problemkonstellationen, wie sie sich aktuell im Bereich der Pflegerobotik abzeichnen, dieser Aufgabe gerecht zu werden:

1. Unsicherheit der Folgenabschätzung: Quelle normativer Unsicherheiten und mithin Gegenstand der ethischen Bewertung ist in der Regel nicht die Technik an sich, sondern deren Rolle in konkreten soziotechnischen Handlungszusammenhängen (Grunwald 2013a, S. 4). Dazu gehören wesentlich die intendierten sowie nicht intendierten Folgen, die mit einem Technikeinsatz verbunden sind und deren moralische Implikationen es ethisch zu klären gilt. In Feldern wie der Pflegerobotik, die sich noch in einem sehr frühen Stadium der Entwicklung befinden und deren Anwendungsmöglichkeiten sich folglich erst unscharf abzeichnen, ist diese konsequenzialistische (folgenorientierte) Herangehensweise mit grundlegenden Problemen konfrontiert - schließlich liegen die Technikfolgen noch weitgehend im Dunkeln, womit auch deren ethische Beurteilung weitgehend ins Leere läuft (was jedoch im Grunde ein Problem unzureichenden prognostischen Wissens und keines der normativen Bewertung an sich darstellt). Die Ethik ist in solchen Fällen entweder auf deontologische Argumentationsmuster - also die Bewertung der Handlung selbst, ungeachtet ihrer Konsequenzen - oder die Formulierung hypothetischer Einsatzszenarien zurückgeworfen, wobei letztere jedoch u. a. aufgrund ihrer weitgehend spekulativen Natur angreifbar erscheinen (Abschn. 8.4).

2. Systemische Effekte: Die soeben beschriebenen Probleme bei der Folgenbeurteilung verkomplizieren sich weiter dadurch, dass die Einbindung neuer Technologien in die Pflege - wie vorliegende Untersuchungen zeigen (Hielscher et al. 2015; Ruiter et al. 2016) - vielfältige und teils subtile, also schwer vorhersehbare Effekte auf die Pflegearbeit haben kann. Selbst eine so harmlos erscheinende Innovation wie die elektronische Patientenakte schafft neue Sachzwänge, ,die das Entscheidungsverhalten der Pflegenden und Ärzte nachhaltig beeinflussen und verändern“ (Manzei 2009, S. 50). Dies dürfte in besonderem Maße für die Pflegerobotik gelten, von der aufgrund ihrer autonomen sowie manipulativen Fähigkeiten besonders weitreichende Folgen (für Pflegehandeln, soziale Interaktionen, berufliche Kompetenzanforderungen etc.) zu erwarten sind. So müssen neben den eigentlichen Arbeitsprozessen nicht selten auch logistische Abläufe sowie das Wohnumfeld angepasst werden, um die autonomen Helfer sinnvoll in die Pflegearbeit einbetten zu können. Die Chancen und Risiken der Pflegerobotik sind deshalb ganzheitlich zu beurteilen: in den Blick zu nehmen ist nicht nur die eigentliche Mensch-Maschine-Interaktion, sondern das Veränderungspotenzial für die soziotechnischen Pflegearrangements insgesamt.

3. Vielgestaltigkeit der Anwendungen: Schließlich kommt erschwerend hinzu, dass die Pflegerobotik keinen monolithischen Bereich bildet, sondern in vielfältige Anwendungsbereiche und Anwendungen zerfällt - korrespondierend $\mathrm{zu}$ der starken Ausdifferenzierung pflegerischer Dienstleistungsbereiche (siehe oben). Das Spektrum der Einsatzmöglichkeiten reicht von einfachen Handhabungs- und Mobilitätshilfen (Esshilfen, Exoskelette etc.) über sozial-interaktive Therapieroboter wie die Robbe Paro bis hin zu mehr oder weniger komplexen Assistenzrobotern, die wiederum für 
sehr unterschiedliche Aufgaben infrage kommen (Gegenstände anreichen, Hebehilfe, Kommunikationsunterstützung etc.). Hinsichtlich Form, Funktion und technischer Komplexität sind diese unterschiedlichen Anwendungen nicht auf einen gemeinsamen Nenner zu bringen. Es liegt auf der Hand, dass sich die Pflegerobotik demzufolge nicht pauschal beurteilen lässt (was u. a. ein Grund dafür ist, weshalb der nivellierende Begriff der Pflegerobotik unter Experten eher kritisch gesehen wird), sondern nur mit einem differenzierten Blick auf die teils sehr verschiedenen Anwendungstypen und Nutzungskontexte.

Zusammengenommen haben die angesprochenen Schwierigkeiten zur Folge, dass die ethischen Debatten zur Pflegerobotik stark um abstrakte begriffliche und vor allem spekulative Erwägungen kreisen, deren empirische Prämissen häufig diffus bleiben. Was normative Orientierungen angeht, lassen sich aus der ethischen Debatte nur einige sehr grobe Anhaltspunkte ableiten. So spitzen sich die ethischen Probleme zu, je stärker autonome Systeme in die personenbezogene Pflege eingreifen. Weitgehende Einmütigkeit herrscht im ethischen Diskurs dahin gehend, dass das Szenario einer voll automatisierten Pflege aufgrund der zentralen pflegerischen Bedeutung zwischenmenschlicher Interaktion moralisch abzulehnen sei (vgl. z. B. Sparrow und Sparrow 2006). Allerdings entspricht dies in der Regel nicht den Zielsetzungen der Entwicklungen, die ja gerade versprechen, durch Entlastung von mühevollen Tätigkeiten mehr Raum für zwischenmenschliche Begegnungen zu schaffen. Viel interessanter wäre deshalb zu erfahren, wie mit den vielfältigen Ambivalenzen umzugehen ist, die beim Einsatz autonomer Assistenzsysteme auftreten können - wichtige Fragen, die sich besonders bei Demenzpatienten stellen, betreffen beispielsweise den möglichen Täuschungscharakter sozialer Therapieroboter oder den Konflikt zwischen dem Schutz der Privatsphäre auf der einen und sicherheitsbezogenen Fürsorgeansprüchen auf der anderen Seite (etwa im Zuge der technischen Überwachung kognitiv eingeschränkter Personen) (Remmers 2016). Konkrete Handreichungen in Form normativer Standards oder Leitlinien, die dazu differenziert Stellung nehmen, liegen jedoch bislang nur in sehr unspezifischer Form vor (vgl. z. B. Manzeschke et al. 2013).

\subsubsection{Upstream engagement als Lösungsansatz?}

Die im letzten Abschnitt diskutierten Bewertungsprobleme sind für die Pflegerobotik nicht spezifisch, sondern verweisen auf eine grundlegende Problematik jeglicher vorausschauenden Technikbewertung und -gestaltung: Eine Folgenabschätzung in der Anfangsphase der Technologieentwicklung (upstream) ist mit einer höchst unsicheren Bewertungsgrundlage konfrontiert, während diese in späteren Phasen mit zunehmender Marktreife (downstream) zwar an Konkretheit gewinnt, wofür dann aber auch kaum noch auf die Technologieentwicklung Einfluss genommen werden kann. Dieses nach dem britischen Technikforscher David Collingridge (1982) benannte Dilemma wirft die Frage auf, wie upstream engagement gelingen kann, wie also zu einem Zeitpunkt auf 
technologische Entwicklungspfade eingewirkt werden kann, zu denen sie noch beeinflussbar und ggf. korrigierbar sind. Dies gilt insbesondere für ethisch hochsensible und kontroverse Bereiche wie die Pflegerobotik, in denen nicht intendierte Folgen unter Umständen besonders gravierende Konsequenzen haben können (andere Beispiele sind die Nanotechnologie oder die Biomedizin).

Ein bereits seit längerem etablierter Ansatz in diesem Zusammenhang ist jener der ELSI-Forschung (ELSI steht für ethical, legal and social implications), der erstmals im Rahmen des Humangenomprojekts in den 1990er Jahren zur Anwendung kam. Das Ziel klassischer ELSI-Forschung ist es, ethischen, rechtlichen und sozialen Aspekten im Rahmen interdisziplinärer Begleitforschung auf den Grund zu gehen, um so möglichen negativen Auswirkungen präventiv begegnen können, etwa durch die Schaffung geeigneter regulativer Rahmenbedingungen. Vor allem biotechnologische Forschungsprogramme wurden entsprechend mit komplementären ELSI-Aktivitäten ausgestattet. Im Laufe der Jahre wurde jedoch auch zunehmend Kritik an diesem Ansatz laut, die sich insbesondere an dessen Begleitforschungscharakter festmachte: Als reines wissenschaftliches Add-on öffentlich geförderter Forschungs- und Entwicklungsprogramme seien ELSI-Forschungen in der Regel zu sehr vom eigentlichen Forschungsgeschehen abgekoppelt (und auch finanziell viel zu stark von diesem abhängig), um dieses kritisch hinterfragen, geschweige denn konstruktiv beeinflussen zu können. ELSI laufe so letztendlich Gefahr, ,eine Art soziales Schmiermittel für eine möglichst nebenwirkungsfreie und reibungslose Umsetzung von Forschung und Technologie“ (Rehmann-Sutter 2011, S. 55 ff.) zu sein.

RRI und andere verwandte Governanceansätze grenzen sich deshalb mehr oder weniger dezidiert von der ELSI-Begleitforschung klassischen Typs ab. ${ }^{2}$ Statt ethische und soziale Aspekte als externe Einflussfaktoren aufzufassen, also als bloße Rahmenbedingungen der Technikentwicklung, sollen sie als Designfaktoren direkt in die Gestaltung der Technologien einfließen. Erforderlich dafür ist offensichtlich eine Verschränkung ethisch-sozialer mit technisch-konstruktiven Fragen, was wiederum eine enge Kollaboration zwischen ELSI-Forschern und wissenschaftlich-technischen Experten bedingt. Von Begleitforschung im eigentlichen Sinne lässt sich dann nicht mehr sprechen - Ziel ist vielmehr ,to increase social-ethical reflexivity within research practices“ (Schomberg 2013, S. 27). Dass die praktische Umsetzung einer solchen „governance from within“ (Fisher et al. 2006) schwierige methodische Fragen aufwirft, liegt auf der Hand. Es sind Disziplinen in einen Austausch zu bringen, die völlig unterschiedlichen Wissenschaftskulturen angehören. Speziell die von einer distanzierend-reflexiven Haltung geprägte Ethik ist herausgefordert, sich auf konkrete technische Fragen und den engen Dialog mit empirisch arbeitenden Disziplinen einzulassen (vgl. Stahl und Coeckelbergh 2016). Die Erwartungen an eine ethische Gestaltung

\footnotetext{
${ }^{2} \mathrm{Zu}$ bemerken ist allerdings, dass die Abgrenzung zwischen der „klassischen“ ELSI-Forschung und der sogenannten Post-ELSI-Forschung alles andere als scharf ist, umso mehr, als auch die ELSI-Ansätze im Laufe der Zeit deutlich weiterentwickelt wurden, vgl. Rehmann-Sutter (2011).
} 
der Technikentwicklung sollten deshalb auch nicht allzu hochgeschraubt werden, wie die Erfahrungen aus dem Bereich der Nanotechnologie zeigen (vgl. Grunwald 2015).

Im Rahmen der angewandten Ethik sind erste Methoden entwickelt worden, die es ermöglichen sollen, ethische Problemstellen emergierender Technologien im Entwicklungsprozess systematisch offenzulegen und bearbeitbar zu machen. Ein Beispiel dafür ist das vom BMBF initiierte MEESTAR-Modell (,Modell zur ethischen Evaluation sozio-technischer Arrangements“, vgl. Manzeschke et al. 2013), das speziell auf neue Technologien im Pflegebereich fokussiert ist. ${ }^{3}$ MEESTAR bietet einen strukturierenden Rahmen ,zur Reflexion und Evaluation konkreter ethischer Fragen und ihrer angemessenen Berücksichtigung im Forschungs-, Entwicklungs- und Anwendungsbereich" innovativer Pflegetechnologien (Manzeschke 2014, S. 11). Im Zentrum stehen sieben ethische Dimensionen (Fürsorge, Autonomie, Sicherheit, Privatheit, Gerechtigkeit, Teilhabe, Selbstverständnis), deren ethische Bedenklichkeit (von völlig unproblematisch bis nicht akzeptabel) es mit Blick auf drei Ebenen (individuell, organisational, gesellschaftlich) einzustufen gilt. Idealerweise geschieht dies im Rahmen eines mehrtägigen interdisziplinären Workshops, in dem diese Aspekte mit konkretem Blick auf eine zu entwickelnde Technologie zu analysieren und zu diskutieren sind.

Das Ziel ist, dass sich die Projektbeteiligten ,ein gemeinsames Bild von der Anwendung“ verschaffen und „zu einem gemeinsamen ethisch begründeten Urteil gelangen, ob und welche Aspekte ihres Projektes einer besonderen Aufmerksamkeit bedürfen“ und wie Lösungsansätze aussehen könnten (Manzeschke 2014; zum konkreten Ablauf vgl. Manzeschke 2015). Das Ziel ist jedoch explizit nicht, zu einer ,generellen und eindeutigen Bewertung" einer bestimmten Anwendung zu kommen. MEESTAR ist damit vor allem als ein Verfahren zu sehen, ,potenzielle Konflikte zu explizieren und Anregung zur Abwägung unterschiedlicher Ansprüche und Interessen zu geben“ (Weber 2015, S. 259). Zentraler normativer Maßstab ist dabei weniger die fachethische Expertise, sondern die Perspektive der an der Entwicklung, dem Einsatz und der Nutzung beteiligten Personen, die natürlich kontrovers und damit aushandlungsbedürftig ist. Der Ethik als Fachdisziplin kommt daher eher die Rolle eines Vermittlers oder Konfliktmoderators als die eines Schiedsrichters zu, womit sich eine These des Wissenschaftssoziologen Alexander Bogners exemplarisch zu bestätigen scheint. Bogner weist darauf hin, dass mit der Ethisierung der Governance - also der wachsenden Bedeutung der Ethik zur Problematisierung von Wissenschafts- und Technikkonflikten - ein grundlegender „Formwandel der Expertise“ einhergeht, der paradoxerweise mit der Abwertung der fachethischen Expertise und gleichzeitig der Aufwertung von deliberativen, partizipativen Verfahren als Legitimationsressource verbunden ist (Bogner 2011). „In diesem Sinne lässt sich Ethisierung als diskursive Entsprechung einer Governance verstehen, die auf Deliberation, Beteiligung und Prozeduralisierung setzt“" (Bogner 2011).

\footnotetext{
${ }^{3}$ Vergleichbare Ansätze, die ebenfalls erst rudimentär ausgearbeitet sind, stammen von van Wynsberghe (2013) sowie Stahl und Coeckelbergh (2016).
} 
RRI ist dafür zweifelsohne ein herausragendes Beispiel, insofern die Ethik hierbei als zentrale ,Triebkraft der Technikentwicklung“ fungieren soll (Bogner et al. 2015, S. 12). Das heißt aber gerade nicht, dass Technology Governance exklusiv in die Hände von professionellen Ethikern sowie anderer Experten gelegt würde. Vielmehr ist die ethische Reflexion von Forschungs- und Innovationsprozessen im Sinne des RRI-Ansatzes auf die möglichst breite Beteiligung und Mitwirkung von Stakeholdern (public engagement) sowie den öffentlichen Diskurs angewiesen, da die Gesellschaft letztlich die einzige legitime Instanz ist, die über die gesellschaftliche Wünschbarkeit einer Entwicklung befinden kann. Insofern ist ein wichtiger Aspekt von RRI, die widerstreitenden Interessen, Ziele und Wertvorstellungen von Akteuren deliberativ offenzulegen, um auf dieser Basis einen kollektiven Reflexionsprozess über wünschenswerte Ziele in Gang setzen zu können (vgl. Lindner et al. 2016, S. 13 f.). Vor diesem Hintergrund wird deutlich, dass ,gesellschaftliche Reflexions- und Kritikkapazitäten“ eine wesentliche Ressource antizipativer Governance sind. Was Wehling (2010) hier für Enhancement-Technologien festgestellt hat, gilt zweifelsohne auch für die Pflegerobotik. Mit Blick auf deren verantwortungsvolle Entwicklung ist folglich festzuhalten, dass neben der bedarfsorientierten Ausrichtung von Forschung und Entwicklung sowie der Berücksichtigung designethischer Fragen auch die diskursiven Rahmenbedingungen von ausschlaggebender Bedeutung sind. $\mathrm{Zu}$ reflektieren ist insbesondere, ob neben robotischen auch nichttechnische Lösungen der Pflegeprobleme infrage kommen oder evtl. gar angemessener sind, und letztlich, inwieweit eine Technisierung der Pflegesituation überhaupt zulässig sein soll. Wird Forschung und Innovation zu innovativen Pflegetechnologien nicht entsprechend in einen breiten gesellschaftlichen Diskurs eingebettet, droht angesichts handfester wirtschaftlicher Sachzwänge und Interessen eine implizite und vorschnelle Festlegung auf bestimmte technologische Entwicklungspfade, was offensichtlich wenig verantwortungsvoll wäre.

\subsection{Mit Robotern gegen den Pflegenotstand? Politische und diskursive Aspekte}

Bereits heute gelten die personellen Bedingungen in der Pflegeversorgung in Deutschland als äußerst prekär, und aufgrund der demografischen Alterung der Bevölkerung ist eine weitere Verschärfung der Situation zu erwarten. Seit einigen Jahren versucht die Bundesregierung deshalb, mit anhaltenden institutionellen Reformen eine finanzielle sowie personelle Schieflage des umlagefinanzierten Pflegesystems abzuwenden und Pflege „demografiefest“ zu gestalten. ${ }^{4}$ Trotz dieser umfassenden Maßnahmen gilt als

\footnotetext{
${ }^{4}$ So wurden in den letzten Jahren mit den sogenannten Pflegestärkungsgesetzen ein Pflegevorsorgefonds eingerichtet, der Pflegebedürftigkeitsbegriff neu definiert (um auch geistige und seelische Beeinträchtigungen besser berücksichtigen zu können) sowie die Beitragssätze zur Pflegeversicherung erhöht (vgl. http://www.pflegestaerkungsgesetz.de). Parallel wird durch eine Pflegeberufereform auch die Pflegeausbildung ganz neu organisiert.
} 
sicher, dass die Pflege auch zukünftig ein „Politikfeld mit stetigem Reformbedarf“ bleiben wird (Rothgang et al. 2015, S. 10). Insofern vermag nicht zu erstaunen, dass nicht nur in Japan, das diesbezüglich als Vorreiter gilt, sondern auch in Deutschland verstärkt Hoffnungen darin gesetzt werden, mittels innovativer Pflegetechnologien die zunehmend angespannte Personalsituation in der Pflege abfedern zu können.

So hat etwa das BMBF erklärt, dass innovative Lösungen gebraucht werden, um „eine bedarfsgerechte und qualitätsvolle Pflege sicherstellen“ zu können. Außerdem solle Deutschland als „Leitanbieter“ im Markt der Pflegetechnologien etabliert werden (BMBF 2015, S. 19). Auch im Koalitionsvertrag von 2013 heißt es, dass „die Entwicklung von Angeboten altersgerechter Begleitung und technischer Unterstützungssysteme“ weiter gefördert werden solle, damit ,ältere und pflegebedürftige Menschen ihren Alltag in der eigenen Wohnung weitgehend selbstbestimmt bewältigen können“ (CDU et al. 2013, S. 84). Im Fokus der deutschen Politik standen diesbezüglich bislang vornehmlich die sogenannten altersgerechten Assistenzsysteme - also intelligente, vernetzte Sensorsysteme (z. B. zur Kommunikation, zur Sturzerkennung oder zum Monitoring von Vitalparametern), auch als Ambient Assisted Living (AAL) bezeichnet -, die seit über zehn Jahren mit verschiedenen Programmen und Maßnahmen strategisch gefördert werden. In den letzten Jahren ist jedoch zu beobachten, dass im Rahmen des BMBF-Forschungsprogramms „Technik zum Menschen bringen“ (BMBF 2015) die Förderaktivitäten zunehmend auf die Pflegerobotik ausgeweitet werden (auch wenn bislang noch eine klare, übergreifende Entwicklungsstrategie zu fehlen scheint, vgl. Kehl 2018, S. 187 ff.). Diese Akzentverschiebung liegt nahe, denn im Unterschied zu AAL-Systemen verfügen Serviceroboter über die Fähigkeit, auch auf physischer Ebene mit Menschen zu interagieren, womit sich deutlich weitreichendere Unterstützungsmöglichkeiten eröffnen als durch die rein virtuell agierenden AAL-Systeme.

Problematisch erscheint allerdings, dass die normativen Herausforderungen, die mit dieser Entwicklung zusammenhängen, auf politischer wie auch gesellschaftlicher Ebene bislang kaum systematisch diskutiert werden. Zwar häufen sich die Medienberichte, die über vereinzelte Entwicklungserfolge (gerne mit Blick auf das fortschrittliche Japan) und mögliche Anwendungsszenarien der Robotik in der Pflege berichten. Das Bild, das dabei von der Robotik und ihren pflegerischen Anwendungsmöglichkeiten gezeichnet wird, ist in der Regel jedoch wenig differenziert, teilweise plakativ und meist stark visionär geprägt. Diskursiver Anknüpfungspunkt ist insbesondere das einflussreiche Leitbild des „artificial companion“ (das übrigens auch in Entwicklerkreisen stark propagiert wird, wie die Pressemeldung des Fraunhofer IPA (2015) zur Lancierung von Care-O-bot 4 zeigt; vgl. auch Krings et al. 2012, S. 41 f.). So wird etwa Care-O-bot, ungeachtet seines prototypischen Entwicklungsstandes, in den Medien gerne als „Universalgenie“ dargestellt, das Getränke servieren, den Tisch decken, Medikamente holen und die Blumen gießen kann (Ringelsiep und Aufmkolk o. J.). Auf diese Weise werden Erwartungen und Vorstellungen geweckt, die weit darüber hinausgehen, was derzeit und auch auf weitere Sicht technisch machbar erscheint. Der humanoide Roboter, der Pflegetätigkeiten direkt am Menschen übernimmt, ist eine ebenso gängige wie wirkmächtige Vision, die 
in Science-Fiction-Filmen wie „Robot \& Frank“ öffentlichkeitswirksam ihren Ausdruck gefunden hat. Die öffentliche Wahrnehmung der Pflegerobotik knüpft damit zum einen an die kulturhistorisch äußerst einflussreiche Figur des Maschinenmenschen an, welche die Entwicklung der Robotik seit jeher geprägt und begleitet hat, sowie zum anderen an neuere Debatten zur maschinellen Superintelligenz, die in transhumanistisch-technikfuturistischen Zirkeln ihren Ausgang genommen hat (vgl. dazu vertiefend Kehl und Coenen 2016, S. 29 ff.).

Eine Folge von all dem ist, dass die öffentliche Diskussion zu den Implikationen der Pflegerobotik hierzulande in stark polarisierter Form geführt wird: Auf der einen Seite gibt es verbreitete Befürchtungen, dass sich Pflege in Deutschland in nicht allzu ferner Zukunft voll automatisiert, also weitgehend maschinell und ohne menschliches Zutun abspielen könnte (z. B. Stösser 2011). Auf der anderen Seite wird argumentiert, dass „Robotik dem aufkommenden Pflegenotstand erfolgreich entgegenwirken“ wird und entsprechende Systeme ,in wenigen Jahren [...] aus dem Pflegealltag nicht mehr wegzudenken sein“werden (z. B. Stiftung Neue Verantwortung 2013). Eine sachliche Auseinandersetzung zu den Potenzialen und Grenzen der Pflegerobotik ist auf Basis derartiger Heils- und Schreckensvisionen schwerlich führbar.

Diese diskursive Gemengelage ist umso kritischer $\mathrm{zu}$ sehen, als gleichzeitig, weitgehend unbemerkt von der Öffentlichkeit, bereits politische Weichenstellungen vorgenommen werden, die einen zukünftigen Einsatz dieser Systeme zumindest wahrscheinlicher machen. Dazu gehört die jüngste Neudefinition des Pflegebedürftigkeitsbegriffs (im Rahmen des Pflegestärkungsgesetzes II, gültig seit 1. Januar 2017), die - um kognitiv und psychisch eingeschränkten Personen besser gerecht werden zu können den Selbstständigkeitsgrad zum zentralen Maßstab für Pflegebedürftigkeit macht (statt dem Hilfebedarf in Minuten). Demnach ist bei der Beurteilung der Selbstständigkeit auch die „Nutzung von Hilfsmitteln“ zu berücksichtigen (vgl. Medizinischer Dienst des Spitzenverbandes Bund der Krankenkassen 2017, S. 37), was laut Arne Manzeschke (2015, S. 266) perspektivisch dazu führen könnte, dass ,,immer mehr Aktivitäten des täglichen Lebens von Pflegebedürftigen durch technische Assistenz unterstützt werden und so das Maß an personaler Hilfe verringert wird“". Parallel dazu wird - wie oben beschrieben die Forschung und Entwicklung zu innovativen Pflegetechnologien politisch vorangetrieben. Auch wenn dabei zunehmend auf eine bedarfsorientierte Vorgehensweise geachtet wird, so lässt sich zumindest für Deutschland sagen, dass die entsprechenden partizipativen Aktivitäten noch auf die Ebene der Technikentwicklung beschränkt sind mit der Konsequenz, dass auf Ebene einzelner Forschungsprogramme zwar das Wie des Technikeinsatzes reflektiert wird, eine übergreifende gesellschaftliche Erörterung des $\mathrm{Ob}$ aber bislang ausgeblieben ist. Was fehlt, ist ein breiter gesellschaftlicher Austausch über wünschenswerte Forschungs- und Entwicklungsziele. 


\subsection{Schluss: Die ambivalente Rolle der Ethik}

Es stellt sich die Frage, inwiefern die philosophische Ethik ihrer Orientierungsfunktion nachzukommen und $\mathrm{zu}$ einer Versachlichung und Neuorientierung der öffentlichen Debatte beizutragen vermag. Immerhin ist die Pflegerobotik inzwischen seit über 10 Jahren Gegenstand intensiver fachethischer Reflexion. Anders als die Nano-Ethik, der es nach Meinung von Grunwald (2015) im Laufe der 2000er Jahre gelungen ist, auf eine „Normalisierung“, also realistischere öffentliche Wahrnehmung der Nanotechnologie hinzuwirken, erweist sich die ethische Debatte zur Pflegerobotik allerdings selber noch weitgehend spekulativem Denken verhaftet. Dies hat nicht nur mit dem philosophischen Hang zu Gedankenspielen zu tun, sondern ganz wesentlich auch mit der mangelnden Verbreitung marktreifer Systeme und dem folglich noch äußerst unsicheren Folgenwissen (Abschn. 8.2.1). Fast notgedrungen sieht sich die philosophische Ethik dadurch, es wurde bereits angesprochen, auf die Diskussion futuristischer Extremszenarien zurückgeworfen, wobei die umfassende Substitution menschlicher Pflegekräfte durch sozial agierende „Pflegeroboter“ (im wahren Sinne des Wortes) ein Fixpunkt der ethischen Debatte darstellt. Beispielhaft sei hier auf den fast schon klassisch zu nennenden Text von Sparrow und Sparrow (2006) mit dem vielsagenden Titel „In the hands of machines“ verwiesen, in dem die Pflege der Zukunft folgendermaßen ausgemalt wird: „,W]e imagine a future aged-care facility where robots reign supreme. In this facility people are washed by robots, fed by robots, monitored by robots, cared for and entertained by robots. Except for their family or community service workers, those within this facility never need to deal or talk with a human being who is not also a resident" (Sparrow und Sparrow 2006, S. 152).

Ähnlich geartete „doom scenarios“ (Coeckelbergh 2016) dienen in ethischen Texten regelmäßig als normative Hintergrundfolie zur Beurteilung möglicher ethischer Implikationen der Pflegerobotik (vgl. z. B. Sparrow 2015; Sharkey und Sharkey 2012b; Borenstein und Pearson 2010) - und zwar ungeachtet dessen, dass zum jetzigen Zeitpunkt völlig unklar ist, ob und wann sich diese weitreichenden Automatisierungsvisionen (resp. die dafür erforderlichen komplexen sozialen Roboter) realisieren lassen. Auf die problematischen Aspekte einer solchen ,spekulativen Ethik“ hat Nordmann (2007) bereits im Kontext nanoethischer Debatten hingewiesen: Es werden Visionen technischer Zukünfte ausgemalt und in einer quasi-deterministischen Zwangsläufigkeit entfaltet, deren Relevanz bei genauerem Hinsehen zumindest fragwürdig erscheint. Reale Herausforderungen, die sich vor allem auf die Gestaltung der technologischen Entwicklung beziehen, geraten dabei aus dem Blickfeld. Damit werden nicht zuletzt auch in der Öffentlichkeit falsche Erwartungen geweckt und wird ein Bild der Pflegerobotik und ihrer Leistungsfähigkeit gezeichnet, das nicht der Realität entspricht.

Zweifelsohne ist die philosophische Beschäftigung mit den erwähnten Szenarien nicht völlig abwegig. So kann sie dabei helfen, begriffliche Grundfragen zu klären und auf normative Grundprobleme aufmerksam zu machen (Grunwald 2010). Im Sinne angewandter Ethik jedoch lässt sich daraus kaum konkretes Handlungs- und Orientierungswissen ableiten, wie es etwa für die Gestaltung neuer oder den Einsatz bestehender Pflegeroboter 
benötigt wird. Symptomatisch ist, dass die entworfenen Anwendungsszenarien ein unterkomplexes Bild der Pflege zeichnen, insofern durch die Fokussierung auf die sozial isolierenden Effekte der Technologie die komplexen soziotechnischen Zusammenhänge der Pflegeinteraktion systematisch unterbelichtet bleiben. Verschiedene Autoren haben deshalb von der Ethik gefordert, ihre Analysen zur Pflegerobotik weniger auf abstrakte Spekulationen zu stützen, sondern stärker am tatsächlichen Gebrauch der Technologien auszurichten (Nylander et al. 2012) und vor allem die dynamischen Wechselwirkungen zwischen Technik und sozialen Nutzungskontexten nicht außer Acht zu lassen (Misselhorn et al. 2013). „Ethics is a journey, and new technologies influence the story“ (Coeckelbergh 2016).

Die Rolle der Ethik im Rahmen antizipativer Governance der Pflegerobotik ist folglich ambivalent: Ohne ethische Orientierung ist die bedarfsorientierte und vor allem verantwortungsvolle Gestaltung der Technologien nicht denkbar, zu beobachten ist jedoch die paradoxe Konsequenz, dass gerade die professionelle ethische Expertise im Zuge der aktuellen Ethisierungsbestrebungen an Relevanz und Einfluss verliert. So wird ethische Reflexion im Rahmen von RRI und verwandten Governanceansätzen einerseits zunehmend prozedural organisiert und partizipativ an fachfremde Akteure delegiert ( $p u b$ lic upstream engagement). Dem öffentlichen Diskurs kommt unter diesen Umständen wie gezeigt besondere Bedeutung zu. Gerade diesbezüglich erweist sich die Fachethik andererseits jedoch nur bedingt hilfreich, insofern sie durch ihre teils spekulative Ausrichtung wenig dazu beiträgt, die tendenziell verzerrte öffentliche Wahrnehmung der Pflegerobotik geradezurücken und sachliche Debatten anzuregen - eher im Gegenteil.

Schließlich offenbart sich hier ein grundlegendes Dilemma des Rufs nach einer verantwortungsvollen Technikgestaltung: Je früher interveniert werden soll, desto unschärfer zeichnen sich die Anwendungsmöglichkeiten, Chancen und Risiken ab, was sachlichen Debatten den Boden entzieht und visionären Spekulationen aller Art Tür und Tor öffnet. Diesen Gap zu überwinden, ist jedoch keine ethische, sondern letztlich eine politische Aufgabe. Klar ist: Die konsequente Förderung bedarfsgerechter Technikentwicklungen allein ist für eine vorausschauende Gestaltung guter Pflege nicht ausreichend. Was es darüber hinaus braucht, ist eine möglichst breit geführte und ergebnisoffene Auseinandersetzung darüber, was wünschenswerte Entwicklungen sind und welche Rolle Automatisierungslösungen im Gesamtkontext der Pflege zukommen soll. Eine solche Debatte findet derzeit weder auf politischer noch auf gesellschaftlicher Ebene statt, wofür nicht zuletzt eine mangelhafte Informationsbasis und teilweise irreführende Technikbilder verantwortlich sind. $\mathrm{Zu}$ wünschen wäre deshalb, dass verstärkt Leuchtturmvorhaben gefördert werden, die sinnvoll erscheinende Pilotapplikationen frühzeitig in die Praxis überführen (vgl. Kehl 2018, S. 205 ff.). Solche „Leuchttürme“ könnten als geschützte Experimentier- und Lernräume fungieren und Pflegeroboter damit nicht nur für die Allgemeinheit anschaulich erfahrbar, sondern auch für die Folgenforschung - insbesondere praxisorientierte ethische Analysen - zugänglich machen. 


\section{Literatur}

BMBF. (2015). Technik zum Menschen bringen; Forschungsprogramm zur Mensch-Technik-Interaktion. https://www.bmbf.de/pub/Technik_zum_Menschen_bringen_Forschungsprogramm.pdf. Zugegriffen: 30. Nov. 2015.

Bogner, A. (2011). Die Ethisierung von Technikkonflikten; Studien zum Geltungswandel des Dissenses. Weilerswist: Velbrück Wiss.

Bogner, A., Decker, M., \& Sotoudeh, M. (2015). Technikfolgenabschätzung und „Responsible Innovation“. In A. Bogner, M. Decker, \& M. Sotoudeh (Hrsg.), Responsible Innovation. Neue Impulse für die Technikfolgenabschätzung? (S. 11-15). Baden-Baden: edition sigma.

Borenstein, J., \& Pearson, Y. (2010). Robot caregivers: Harbingers of expanded freedom for all? Ethics and Information Technology, 12, 277-288. https://doi.org/10.1007/s10676-010-9236-4.

Bundesregierung. (2016). Siebter Bericht zur Lage der älteren Generation in der Bundesrepublik Deutschland; Sorge und Mitverantwortung in der Kommune - Aufbau und Sicherung zukunftsfähiger Gemeinschaften und Stellungnahme der Bundesregierung. http://www.demografie-portal.de/SharedDocs/Downloads/DE/BerichteKonzepte/Bund/Siebter-Altenbericht. pdf?_blob=publicationFile\&v=2. Zugegriffen: 03. Sept. 2018.

CDU, CSU, \& SPD. (2013). Deutschlands Zukunft gestalten; Koalitionsvertrag zwischen CDU, CSU und SPD. https://www.bundesregierung.de/Content/DE/_Anlagen/2013/2013-12-17-koalitionsvertrag.pdf?_blob=publicationFile. Zugegriffen: 03. Sept. 2018.

Coeckelbergh, M. (2016). Care robots and the future of ICT-mediated elderly care; A response to doom scenarios. AI \& Society, 31, 455-462. https://doi.org/10.1007/s00146-015-0626-3.

Collingridge, D. (1982). The social control of technology. London: Pinter.

Decker, M. (2013). Robotik. In A. Grunwald (Hrsg.), Handbuch Technikethik. Stuttgart: Metzler.

Decker, M., \& Weinberger, N. (2015). Was sollen wir wollen - Möglichkeiten und Grenzen der bedarfsorientierten Technikentwicklung. In R. Weidner, T. Redlich, \& J. P. Wulfsberg (Hrsg.), Technische Unterstützungssysteme (S. 19-29). Berlin: Springer.

Derpmann, S., \& Compagna, D. (2009). Verfahren partizipativer Technikentwicklung. Working Papers kultur- und techniksoziologische Studien (S. 1-33). Duisburg: Universität Duisburg-Essen.

Dibelius, O., Uzarewicz, C., \& Tesch-Römer, C. (2006). Pflege von Menschen höherer Lebensalter. Stuttgart: Kohlhammer.

Elsbernd, A., Lehmeyer, M. S., Schilling, M. U., Warendorf, K., \& Wu, M. S. J. (2014). Bedarfsgerechte technikgestützte Pflege in Baden-Württemberg-Technologien und Dienstleistungen für ein selbstbestimmtes Leben im Alter.

Elsbernd, A., Lehmeyer, S., \& Schillingen, U. (2015). Pflege und Technik-Herausforderungen an ein interdisziplinäres Forschungsfeld. Pflege \& Gesellschaft, 20, 67-76.

Evans, J. M. (1994). HelpMate: An autonomous mobile robot courier for hospitals IROS '94. Proceedings of the IEEE/RSJ/GI international conference on intelligent robots and systems. Institute of Electrical and Electronics Engineers; Available from IEEE Service Center, New York, Piscataway, S. 1695-1700.

Fisher, E., Mahajan, R. L., \& Mitcham, C. (2006). Midstream Modulation of Technology: Governance from within. Bulletin of Science, Technology \& Society, 26, 485-496. https://doi. org/10.1177/0270467606295402.

Fraunhofer, IPA. (2015). Presseinformation: Roboter als vielseitiger Gentleman, Stuttgart. https:// www.ipa.fraunhofer.de/de/presse/presseinformationen/2015-01-15_roboter-als-vielseitigergentleman.html. Zugegriffen: 03. Sept. 2018. 
Görres, S., \& Friesacher, H. (2005). Der Beitrag der Soziologie für die Pflegewissenschaft, Pflegetheorien und Pflegemodelle. In K. R. Schroeter \& T. Rosenthal (Hrsg.), Soziologie der Pflege. Grundlagen, Wissensbestände und Perspektiven. Weinheim: Juventa-Verl.

Grunwald, A. (2010). From speculative nanoethics to explorative philosophy of nanotechnology. Nanoethics, 4, 91-101.

Grunwald, A. (2013a). Einleitung. In A. Grunwald (Hrsg.), Handbuch Technikethik (S. 1-11). Stuttgart: Metzler.

Grunwald, A. (2013b). Ethische Aufklärung statt Moralisierung. Zur reflexiven Befassung der Technikfolgenabschätzung mit normativen Fragen. In A. Bogner (Hrsg.), Ethisierung der Technik - Technisierung der Ethik. Der Ethik-Boom im Lichte der Wissenschafts- und Technikforschung (S. 232-246). Baden-Baden: Nomos.

Grunwald, A. (2015). Fünfzehn Jahre Ethik zur Nanotechnologie - was wurde bewirkt? In M. Maring (Hrsg.), Vom Praktisch-Werden der Ethik in interdisziplinärer Sicht: Ansätze und Beispiele der Institutionalisierung, Konkretisierung und Implementierung der Ethik (S. 281-297). Karlsruhe: KIT Scientific Publishing.

Hielscher, V., Nock, L., \& Kirchen-Peters, S. (2015). Technikeinsatz in der Altenpflege; Potenziale und Probleme in empirischer Perspektive. Baden-Baden: Nomos.

Hülsken-Giesler, M. (2008). Der Zugang zum Anderen; Zur theoretischen Rekonstruktion von Professionalisierungsstrategien pflegerischen Handelns im Spannungsfeld von Mimesis und Maschinenlogik. Göttingen: V \& R Unipress.

Kehl, C., \& Coenen, C. (2016). Technologien und Visionen der Mensch-Maschine-Entgrenzung; Sachstandsbericht zum TA-Projekt „Mensch-Maschine-Entgrenzungen: zwischen künstlicher Intelligenz und Human Enhancement " Berlin: Büro für Technikfolgen-Abschätzung beim Deutschen Bundestag. http://www.tab-beim-bundestag.de/de/pdf/publikationen/berichte/TAB-Arbeitsbericht-ab167.pdf. Zugegriffen: 03. Sept. 2018.

Kehl, C. (2018). Robotik und assistive Neurotechnologien in der Pflege-gesellschaftliche Herausforderungen. Berlin: Büro für Technikfolgen-Abschätzung beim Deutschen Bundestag. http:// www.tab-beim-bundestag.de/de/pdf/publikationen/berichte/TAB-Arbeitsbericht-ab177.pdf. Zugegriffen: 03. Sept. 2018.

Krings, B.-J., Fleischer, T., Schippl, J., \& Weinberger, N. (2012). ITA-Monitoring „Serviceroboter in Pflegearrangements“; Pre-Print: 4.12.2012. http://www.itas.kit.edu/pub/v/2012/epp/krua12pre01.pdf. Zugegriffen: 07. Jan. 2016.

Lindner, R., Goos, K., Güth, S., Som, O., \& Schröder, T. (2016). „Responsible Research and Innovation" als Ansatz, für die Forschungs-, Technologie- und Innovationspolitik - Hintergründe und Entwicklungen. Berlin: Büro für Technikfolgen-Abschätzung beim Deutschen Bundestag.

Loh, J. (2017). Roboterethik. Über eine noch junge Bereichsethik. Information Philosophie, 1, 20-33.

Manzei, A. (2009). Neue betriebswirtschaftliche Steuerungsformen im Krankenhaus: wie durch die Digitalisierung der Medizin ökonomische Sachtwänge in der Pflegepraxis entstehen. Pflege und Gesellschaft, 14, 38-53.

Manzeschke, A. (2014). Ethische Herausforderungen technologischen Wandels. Informationsdienst Altersfragen, 41, 10-18.

Manzeschke, A. (2015). MEESTAR: Ein Modell angewandter Ethik im Bereich assistiver Technologien. In K. Weber (Hrsg.), Technisierung des Alltags. Beitrag für ein gutes Leben? Stuttgart: Steiner.

Manzeschke, A., Weber, K., Rother, E., \& Fangerau, H. (2013). Ergebnisse der Studie «Ethische Fragen im Bereich Altersgerechter Assistenzsysteme». https://www.technik-zum-menschen-bringen.de/dateien/service/broschuere-ethische-fragen-altersgerechte-assistenzsysteme.pdf/download. Zugegriffen: 03. Sept. 2018.

Medizinischer Dienst des Spitzenverbandes Bund der Krankenkassen. (2017). Richtlinien des GKV-Spitzenverbandes zur Feststellung der Pflegebedürftigkeit nach dem XI. Buch des Sozial- 
gesetzbuches. https://www.mds-ev.de/fileadmin/dokumente/Publikationen/SPV/Begutachtungsgrundlagen/17-07-17_BRi_Pflege.pdf. Zugegriffen: 03. Sept. 2018.

Misselhorn, C., Pompe, U., \& Stapleton, M. (2013). Ethical considerations regarding the use of social robots in the fourth age. GeroPsych: The Journal of Gerontopsychology and Geriatric Psychiatry, 26, 121-133.

Neuber-Pohl, C. (2017). Das Pflege- und Gesundheitspersonal wird knapper. Berufsbildung in Wissenschaft und Praxis, 46, 4-5.

Nordmann, A. (2007). If and then: A critique of speculative nanoethics. Nanoethics, 1, 31-46. https://doi.org/10.1007/s11569-007-0007-6.

Nylander, S., Ljungblad, S., \& Jimenez Villareal, J. (2012). A complementing approach for identifying ethical issues in care robotics - grounding ethics in practical use. 2012 IEEE RO-MAN: the $21^{\text {st }}$ IEEE International Symposium on Robot and Human Interactive Communication. Paris, France, 9-13 September 2012. IEEE, Piscataway, S. 797-802.

Östlund, B., \& Frennert, S. (2014). Seven matters of concern of social robotics and older people. International Journal of Social Robotics, 6, 299-310.

Piekenbrock, D. (2018). Bedarf. In Gabler Wirtschaftslexikon. https://wirtschaftslexikon.gabler.de/ definition/bedarf-28254. Zugegriffen: 11. Mai 2018.

Rehmann-Sutter, C. (2011). Gesellschaftliche, rechtliche und ethische Implikationen der Biomedizin; Zu der Rolle und den Aufgaben von ELSI-Begleitforschung. In S. Dickel, M. Franzen, \& C. Kehl (Hrsg.), Herausforderung Biomedizin. Gesellschaftliche Deutung und soziale Praxis (S. 49-66). Bielefeld: Transcript.

Remmers, H. (2016). Ethische Implikationen der Nutzung altersgerechter technischer Assistenzsysteme; Expertise zum Siebten Altenbericht der Bundesregierung. https://www.siebter-altenbericht.de/fileadmin/altenbericht/pdf/Expertise_Remmers.pdf. Zugegriffen: 03. Sept. 2018.

Ringelsiep, M., \& Aufmkolk, T. (o.J.). Care-O-bot - Pfleger aus Stahl. https://www.planet-wissen.de/technik/computer_und_roboter/roboter_mechanische_helfer/pwiecareobotpflegerausstahl100.html. Zugegriffen: 03. Sept. 2018.

Rothgang, H., Kalwitzki, T., Müller, R., Runte, R., \& Unger, R. (2015). BARMER GEK Pflegereport 2015; Schwerpunktthema: Pflegen zu Hause. https://www.barmer.de/blob/36042/73f4ded2ea20652834aeedbb7c2bc16a/data/pdf-barmer-gek-pflegereport-2015.pdf. Zugegriffen: 03 . Sept. 2018.

Ruiter, H-P. de., Liaschenko, J., \& Angus, J. (2016). Problems with the electronic health record. Nursing Philosophy : An International Journal for Healthcare Professionals , 17, 49-58. https:// doi.org/10.1111/nup.12112.

Sharkey, A., \& Sharkey, N. (2012a). Granny and the robots: Ethical issues in robot care for the elderly. Ethics Inf Technol, 14, 27-40. https://doi.org/10.1007/s10676-010-9234-6.

Sharkey, N., \& Sharke, A. (2012b). The eldercare factory. Gerontology, 58, 282-288. https://doi. org/10.1159/000329483.

Shibata, T. (2012). Therapeutic seal robot as biofeedback medical device: Qualitative and quantitative evaluations of robot therapy in dementia care. Proceedings of the IEEE, 100, 2527-2538.

Sparrow, R. (2015). Robots in aged care; A dystopian future? AI \& Society. https://doi. org/10.1007/s00146-015-0625-4.

Sparrow, R., \& Sparrow, L. (2006). In the hands of machines?; the future of aged care. Minds \& Machines, 16, 141-161. https://doi.org/10.1007/s11023-006-9030-6.

Stahl, BC., \& Coeckelbergh, M. (2016). Ethics of healthcare robotics; Towards responsible research and innovation. Robotics and Autonomous Systems, 86, 152-161.

Stiftung Neue Verantwortung. (2013). Mit Robotern gegen den Pflegenotstand; Policy Brief. http:// www.ndws.de/wp-content/uploads/2015/02/Beck2013MRG.pdf. Zugegriffen: 25. Sept. 2015.

Stösser, Av. (2011). Roboter als Lösung für den Pflegenotstand?; Ethische Fragen. ARCHIV für Wissenschaft und Praxis der sozialen Arbeit, 3, 99-107. 
Sutcliffe, H. (2013). A report on responsible research \& innovation. http://ec.europa.eu/research/ science-society/document_library/pdf_06/rri-report-hilary-sutcliffe_en.pdf. Zugegriffen: 05 . Dez. 2017.

Topping, M. (2000). An overview of the development of Handy 1, a Rehabilitation robot to assist the severely disabled. Artificial Life and Robotics, 4, 188-192. https://doi.org/10.1007/ bf02481173.

van Wynsberghe, A. (2013). Designing robots for care: Care centered value-sensitive design. Science and Engineering ethics, 19, 407-433. https://doi.org/10.1007/s11948-011-9343-6.

Von Schomberg, R. (2013). A vision of responsible research and innovation. In R. Owen, J. Bessant, \& M. Heintz (Hrsg.), Responsible innovation. Managing the responsible emergence of science and innovation in society. London: Wiley.

Weber, K. (2015). MEESTAR: Ein Modell zur ethischen Evaluierung sozio-technischer Arrangements in der Pflege- und Gesundheitsversorgung. In K. Weber (Hrsg.), Technisierung des Alltags. Beitrag für ein gutes Leben? Stuttgart: Steiner.

Wehling, P., (2010). „Anticipatory Governance“ von Technisierungsprojekten?; Möglichkeiten und Grenzen am Beispiel von Enhancement-Technologien. In G. Aichholzer, A. Bora, S. Bröchler, M. Decker, \& M. Latzer (Hrsg.), Technology Governance. Der Beitrag der Technikfolgenabschätzung (S. 155-162). Berlin: sigma.

Weinberger, N., \& Decker., M. (2015). Technische Unterstützung für Menschen mit Demenz?; Zur Notwendigkeit einer bedarfsorientierten Technikentwicklung. Technikfolgenabschätzung Theorie und Praxis, 24, 36-45.

Dr. Christoph Kehl studierte Umweltnaturwissenschaften (ETH Zürich) und Philosophie (FU Berlin) und promovierte im Bereich der Wissenschafts- und Technikforschung. Seit 2012 ist er wissenschaftlicher Mitarbeiter des Büros für Technikfolgen-Abschätzung beim Deutschen Bundestag (TAB) und in diesem Zusammenhang an verschiedenen Untersuchungen beteiligt, die sich mit dem digitalen Wandel und seinen gesellschaftlichen Implikationen beschäftigen. U. a. arbeitete er an einem TAB-Bericht zu „Technologien und Visionen der Mensch-Maschine-Entgrenzung“ mit (Arbeitsbericht Nr. 167, erschienen 2016) und leitete ein TAB-Projekt zum Thema „Robotik und assistive Neurotechnologien in der Pflege - gesellschaftliche Herausforderungen“ (Arbeitsbericht Nr. 177, erschienen 2018).

Open Access Dieses Kapitel wird unter der Creative Commons Namensnennung 4.0 International Lizenz (http://creativecommons.org/licenses/by/4.0/deed.de) veröffentlicht, welche die Nutzung, Vervielfältigung, Bearbeitung, Verbreitung und Wiedergabe in jeglichem Medium und Format erlaubt, sofern Sie den/die ursprünglichen Autor(en) und die Quelle ordnungsgemäß nennen, einen Link zur Creative Commons Lizenz beifügen und angeben, ob Änderungen vorgenommen wurden.

Die in diesem Kapitel enthaltenen Bilder und sonstiges Drittmaterial unterliegen ebenfalls der genannten Creative Commons Lizenz, sofern sich aus der Abbildungslegende nichts anderes ergibt. Sofern das betreffende Material nicht unter der genannten Creative Commons Lizenz steht und die betreffende Handlung nicht nach gesetzlichen Vorschriften erlaubt ist, ist für die oben aufgeführten Weiterverwendungen des Materials die Einwilligung des jeweiligen Rechteinhabers einzuholen.

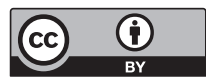

